\title{
PRIMES IN ARITHMETIC PROGRESSIONS TO LARGE MODULI. III
}

\author{
E. BOMBIERI, J. B. FRIEDLANDER, AND H. IWANIEC
}

\section{INTRODUCTION}

For $q$ a positive integer and $a$ an integer prime to $q$, we let

$$
\pi(x ; q, a)=\sum_{\substack{p \leq x \\ p \equiv a(\bmod q)}} 1
$$

count those primes up to $x$ in the arithmetic progression $a$ modulo $q$. One expects these arithmetic progressions to contain their due proportion of primes, namely

$$
\pi(x ; q, a) \sim \pi(x) / \phi(q),
$$

where $\phi$ is Euler's function and $\pi(x)$ counts all primes up to $x$. Indeed, (1.1) is known to hold, but only under the proviso that $q$ grow more slowly than some fixed power of $\log x$ (Siegel-Walfisz theorem). Yet, for many applications, it is important to weaken this restriction. The assumption of the Generalized Riemann Hypothesis (GRH) yields (1.1) in the much wider range $q<x^{1 / 2} / \log ^{2+\varepsilon} x$.

It is often sufficient, in particular for applications of the sieve to the study of integers of the form $p-a$, to have access to such results in an averaged form and the Bombieri-Vinogradov theorem [2] gives such a bound, in that for any $A>0$ and some $B=B(A)$,

$$
\sum_{\substack{q<x^{1 / 2} \log ^{-B} x \\(q, a)=1}}|\pi(x ; q, a)-\pi(x) / \phi(q)| \ll_{A} x \log ^{-A} x .
$$

This deals with essentially the same range of $q$ as can be treated conditionally under GRH for individual progressions.

For significantly larger $q$, the asymptotic formula is not known to hold, even in the above averaged sense, but there do follow by sieve methods upper bounds of the expected order of magnitude under the very mild restriction $q<x^{1-\varepsilon}$. Known as Brun-Titchmarsh theorems, these bounds hold both for the individual

Received by the editors May 24, 1988.

1980 Mathematics Subject Classification (1985 Revision). Primary 11 N13.

The second author was supported in part by NSERC grant A5123. 
sum $\pi(x ; q, a)$ and, with better constants, for averages of the type occurring on the left-hand side of (1.2). Each of the above types of results enjoys some advantages over the others, and all find application to a wide range of problems both in prime number theory and further afield.

In this paper, we prove the following.

Main Theorem. Let $a \neq 0$ be an integer and $A>0,2 \leq Q \leq x^{3 / 4}$ be reals. Let $\mathscr{Q}$ be the set of all integers $q$, prime to $a$, from an interval $Q^{\prime}<q \leq Q$. Then

$$
\begin{aligned}
& \sum_{q \in \mathscr{Q}}\left|\pi(x ; q, a)-\frac{\pi(x)}{\phi(q)}\right| \\
& \quad \leq\left\{K\left(\theta-\frac{1}{2}\right)^{2} x L^{-1}+O\left(x L^{-3}(\log \log x)^{2}\right)\right\} \sum_{q \in \mathscr{Q}} \frac{1}{\phi(q)}+O\left(x L^{-A}\right),
\end{aligned}
$$

where $\theta=\log Q / \log x, L=\log x, K$ is absolute, the first implied constant in $O$ depends only on $A$, and the second one on $a$ and $A$.

This result provides a continuous transition from Bombieri-Vinogradov type theorems to Brun-Titchmarsh type theorems. Thus, on the one hand, it gives the asymptotic formula for $Q=x^{1 / 2} g(x)$ with any $g(x)$ satisfying $\log g(x)=$ $o(\log x)$, thereby refining the result in [4]. On the other hand, it gives for fixed $\theta>\frac{1}{2}$ Brun-Titchmarsh theorems on average, where one may come arbitrarily close to the expected constant by taking $\theta$ sufficiently close to $\frac{1}{2}$. This is in contrast to earlier theorems of this type. The most recent, due to Rousselet [11], requires .48 in place of $K\left(\theta-\frac{1}{2}\right)^{2}$ for $\theta$ near $\frac{1}{2}$.

The arguments used in the proof of the main theorem overlap very heavily the arguments of our earlier paper [4]. We have decided to assume knowledge of [4]. To do otherwise would be to enormously expand the length of this paper.

For economy of presentation, we have not attempted to determine an explicit value for the constant $K$. The determination of a reasonable admissible value for this constant seems to offer no difficulty in principle, and we hope to return to this at some future time. This is worthwhile in view of the number of applications these theorems have; see, for example, $[1,5,6]$. Since we do not specify the constant now, it suffices to deal with the case $\theta \leq \frac{1}{2}+\eta$, where, say, $\eta=10^{-4}$. Also, in view of the classical Bombieri-Vinogradov theorem [2], we are able to assume that $Q>x^{1 / 2} L^{-B}$, with some fixed $B$. For $\theta$ in this interval, we expect 36 to be an admissible value for $K$.

\section{Mean Value theorems}

We shall make use of a number of mean value theorems for bilinear and trilinear forms, to a large extent the same as those in [4].

We begin by recalling some assumptions from [4], namely $\left(A_{1}\right)$ [4, p. 364] and $\left(A_{2}\right)$ and $\left(A_{3}\right)$ [4, p. 371]. Here we shall use $\left(A_{1}\right)$ and $\left(A_{2}\right)$ but modify 
$\left(\mathrm{A}_{3}\right)$ to
$\left(A_{3}^{c}\right)$
$\beta_{n}=0$ unless $\left(n, P\left(L^{c}\right)\right)=1$,

where, as usual, $P(y)$ is the product of all primes less than $y$.

Theorem 1. Let $M N=x, \alpha=\left(\alpha_{m}\right), m \sim M, \beta=\left(\beta_{n}\right), n \sim N, a \neq 0$, $\varepsilon>0$, and $A>0$. Suppose $\beta$ satisfies $\left(\mathrm{A}_{1}\right)$ and $\left(\mathrm{A}_{2}\right)$. Then there exist constants $c$ and $A_{0}$ such that provided $\beta$ satisfies $\left(\mathrm{A}_{3}^{\mathrm{c}}\right)$ and

$$
Q^{2} x^{-1} L^{A_{0}}<N<x^{5 / 6-2 \varepsilon} Q^{-4 / 3},
$$

then

$$
\sum_{\substack{q \sim Q \\(q, a)=1}}|\Delta(\alpha * \beta ; q, a)| \ll\|\alpha\|\|\beta \tau\| x^{1 / 2} L^{-A} .
$$

Here $\tau$ denotes the divisor function, so $\beta \tau=\left(\beta_{n} \tau(n)\right),\|\|$ denotes the $l_{2}$ norm, $m \sim M$ means $\frac{M}{2}<m \leq M, c$ depends on $A$ and $B, A_{0}$ depends on $A$, and the implied constant may depend on $\varepsilon, a, A, B$, and $B_{1} . \Delta$ is defined in $[4$, p. 363].

Proof. This is a slight modification of Theorem 1 of [4], which was proved as Theorem 3 in [3]. The assumption $\left(A_{3}^{c}\right)$ rather than $\left(A_{3}\right)$ was actually what was required in that proof. The only other difference is the weaker lower bound for $N$ in (2.1), leading to the appearance of $\tau$ in (2.2). This may be justified by a slight improvement of the estimate (10.6) of [3, p. 232] as follows. As there, the terms on the diagonal are majorized by

$$
Q^{2} \sum_{\left(n_{1}, n_{2}\right)=1}\left|\beta_{n_{1}} \beta_{n_{2}}\right|^{2} \sum_{\substack{n_{3}, n_{4} \sim N \\ h_{1}\left(n_{2}-n_{1}\right) n_{3} n_{4}=h_{2}\left(n_{4}-n_{3}\right) n_{1} n_{2}}} \sum_{\substack{1 \leq h_{1}, h_{2} \leq H \\ \text {. }}} 1 .
$$

Given $h_{2}, n_{1}, n_{2}$, then $n_{3} n_{4} \mid h_{2} n_{1} n_{2}$. Thus, the number of triples $\left(h_{1}, n_{3}, n_{4}\right)$ is $\leq \tau^{2}\left(h_{2}\right) \tau^{2}\left(n_{1}\right) \tau^{2}\left(n_{2}\right)$ so that $(2.3)$ is majorized by

$$
Q^{2} \sum_{\substack{h_{2} \leq H \\\left(n_{1}, n_{2}\right)=1}}\left|\beta_{n_{1}} \beta_{n_{2}}\right|^{2} \tau^{2}\left(h_{2}\right) \tau^{2}\left(n_{1}\right) \tau^{2}\left(n_{2}\right) \ll Q^{2} H L^{3}\|\beta \tau\|^{4} .
$$

Using the more precise smoothing function of [4, Lemma 9, Corollary] rather than that of [3, Lemma 2], we may choose $H=M^{-1} Q^{2} L^{A_{1}}$ for some $A_{1}$ depending on $A$. This completes the proof of Theorem 1 .

Assume Theorems 2-5 of [4]. (Theorem $5^{*}$ of [4] will not be used.) We remark that as in Theorem 1 any use of $\left(A_{3}\right)$ in these later theorems may be weakened to the assumption of $\left(A_{3}^{c}\right)$ for suitable $c$. However, this remark is not needed for the proof of our main theorem (although it could be used to slightly economize our presentation). 


\section{COMBINATORIAL PREPARATIONS}

In this section, we apply combinatorial arguments to reduce the proof of our main theorem to the estimation of sums that fall within the scope of the mean value theorems of the preceding section.

As compared to [4], we use the identity of Linnik rather than that of HeathBrown. This will prove significant for the determination of numerical values for $K$.

Let

$$
E(x, \mathscr{Q})=\sum_{q \in \mathscr{Q}}\left|\sum_{\substack{n \sim x \\ n \equiv a(q)}}^{(z)} \frac{\Lambda(n)}{\log n}-\frac{1}{\phi(q)} \sum_{\substack{n \sim x \\(n, q)=1}}^{(z)} \frac{\Lambda(n)}{\log n}\right|,
$$

where $\sum^{(z)}$ restricts the summation to $n$ with $(n, P(z))=1$.

We choose $z=x^{5 / 6-2 \varepsilon} Q^{-4 / 3}$ and note that $x^{1 / 7}<z \leq x^{1 / 6-\varepsilon}$ since $x^{1 / 2} L^{-B}<Q<x^{1 / 2+1 / 56}$.

Lemma 1. For $n>1$, we have

$$
\frac{\Lambda(n)}{\log n}=\sum_{j=1}^{\infty} \frac{(-1)^{j-1}}{j} t_{j}(n),
$$

where

$$
t_{j}(n)=\sum_{k=0}^{j}(-1)^{j-k}\left(\begin{array}{l}
j \\
k
\end{array}\right) \tau_{k}(n)
$$

is the number of decompositions of $n$ as the product of precisely $j$ integers greater than one.

Proof. This is Linnik's identity [10].

The sum over $j$ always terminates and, if $(n, P(z))=1$, then $t_{j}(n)=0$ for $j>\log n / \log z$. By Lemma 1 ,

$$
E(x, \mathscr{Q}) \leq \sum_{j=1}^{6} \frac{1}{j} E_{j}(x, \mathscr{Q}),
$$

where $E_{j}$ is a sum similar to $E$ but with $\Lambda(n) / \log n$ replaced by $t_{j}(n)$. It clearly suffices to prove that, for $1 \leq j \leq 6$, and for $\mathscr{Q}$ contained in $(Q / 2, Q]$, (3.2)

$$
E_{j}(x, \mathscr{Q}) \leq\left\{K_{j}\left(\theta-\frac{1}{2}\right)^{2} x L^{-1}+O\left(x L^{-3}(\log \log x)^{2}\right)\right\} \sum_{q \in K^{\prime}} \frac{1}{\phi(q)}+O\left(x L^{-A}\right),
$$

and the main theorem then follows with

$$
K=\sum_{j=1}^{6} \frac{1}{j} K_{j} .
$$

Throughout the proof we shall be dealing with various sums $\sum_{n_{1}, \ldots, n_{1}} 1$. These sums will always be restricted to $n_{1}, \ldots, n_{j}$ that satisfy the constraints 
$n_{1} \cdots n_{j} \sim x, n_{1} \geq z, \ldots, n_{j} \geq z$. Any additional constraints will be specifically mentioned but these will not.

We also adopt the convention that all implied constants are absolute, unless specific mention is made to the contrary, with the unique exception that in error terms of the form $O\left(x L^{-A}\right)$ the implied constants $(O$ or $\ll)$ may depend on $a$ and $A$.

\section{THE CASES $1 \leq j \leq 3$}

Let

$$
\Delta_{j}(x, z ; q, a)=\sum_{n_{1} \cdots n_{j} \equiv a(q)}^{(z)} 1-\frac{1}{\phi(q)} \sum_{\left(n_{1} \cdots n_{j}, q\right)=1}^{(z)} 1 .
$$

In [4], we dealt with the cases $j \leq 3$ by using ([4, Lemma 2]) results for the divisor function $\tau_{j}$ in arithmetic progressions. These results cannot be immediately applied to $\Delta_{j}$ because of the condition $\left(n_{i}, P(z)\right)=1$ and in [4] this condition was removed via a sieve-theoretic fundamental lemma. In the present case, our choice of $z$ is much larger and we first reduce to $\left(n_{i}, P(w)\right)=1$ with

$$
w=Q^{2} x^{-1} L^{A_{0}}
$$

(where $A_{0}$ is as in Theorem 1) by means of the Buchstab identity:

$$
\Delta_{j}(x, z ; q, a)=\Delta_{j}(x, w ; q, a)-\sum_{w \leq p<z} \Delta_{j p}(x, p ; q, a),
$$

where $\Delta_{j}(x, w ; q, a)$ changes only the condition $\left(n_{i}, P(z)\right)=1$ to $\left(n_{i}, P(w)\right)$ $=1$ but keeps the condition $n_{i} \geq z$, and

$$
\Delta_{j p}(x, p ; q, a)=\sum_{n_{1} \cdots n_{1} \equiv a(q)} 1-\frac{1}{\phi(q)} \sum_{\left(n_{1} \cdots n_{j}, q\right)=1} 1,
$$

where both sums are also restricted by the conditions $n_{1} \cdots n_{j} \equiv 0(p)$, $\left(n_{1} \cdots n_{j}, P(p)\right)=1$.

We consider the contribution to $E_{j}$ from the $\Delta_{j p}$. By an inclusion-exclusion argument, the condition $n_{1} \cdots n_{j} \equiv 0(p)$ may be reduced to the simultaneous conditions $n_{i_{1}} \equiv 0(p), \ldots, n_{i_{k}} \equiv 0(p)$ for the various nonempty subsets of indices $\left\{i_{1}, \ldots, i_{k}\right\}$ of $\{1, \ldots, j\}$. The corresponding sums with these conditions are now, after a dyadic subdivision of the ranges of $p$ and the $n_{i}$, in a form to which Theorem 1 applies. This is due to our choices of $z$ and $w$. The result is

$$
\sum_{q \in \kappa^{\prime}}\left|\sum_{w \leq p<z} \Delta_{j p}(x, p ; q, a)\right| \ll x L^{-. t} .
$$

It now remains to treat $\Delta_{j}(x, w ; q, a)$. As in [4], we are now in a position to use the fundamental lemma but $w$ is still somewhat large, and we require more care than previously. In particular, we shall require the fundamental lemma in the following form, which is slightly stronger than [4, Lemma 3]. 
Lemma 2. Let $s \geq 3$ and $D=w^{s}$. There exist two sequences $\left\{\lambda_{d}^{+}\right\}_{d \leq D}$ and $\left\{\lambda_{d}^{-}\right\}_{d \leq D}$ such that

$$
\begin{aligned}
& \left|\lambda_{d}^{ \pm}\right| \leq 1 \quad \text { for } d \mid P(w), \quad \lambda_{d}^{ \pm}=0 \text { otherwise, } \\
& \left(\lambda^{-} * 1\right)(n)=\left(\lambda^{+} * 1\right)(n)=1 \quad \text { if }(n, P(w))=1, \\
& \left(\lambda^{-} * 1\right)(n) \leq 0 \leq\left(\lambda^{+} * 1\right)(n) \quad \text { if }(n, P(w))>1,
\end{aligned}
$$

and, for any multiplicative function $\omega(d)$ with $0 \leq \omega(p) \leq \min \{\kappa, p-1\}$ we have

$$
\sum_{d \leq D} \lambda_{d}^{ \pm} \frac{\omega(d)}{d}=\prod_{p \mid P(w)}\left(1-\frac{\omega(p)}{p}\right)\left\{1+O\left(s^{-s}\right)\right\},
$$

the constant implied in $O$ depending on $\kappa$ alone.

Proof. See [7] or [9].

We apply Lemma 2 in the manner of [4, pp. 365-366]. We have

$$
\pm \Delta_{j}(x, w ; q, a) \leq \pm \sum_{(d, q)=1}\left\{\lambda_{d}^{ \pm} T_{d}(q, a)-\lambda_{d}^{\mp} \frac{T_{d}(q)}{\phi(q)}\right\}
$$

and hence

$$
\Delta_{j}(x, w ; q, a) \leq \sum_{\substack{(d, q)=1 \\ d \leq D}}\left|T_{d}(q, a)-\frac{1}{\phi(q)} T_{d}(q)\right|+\frac{1}{\phi(q)}\left|\sum_{(d, q)=1}\left(\lambda_{d}^{+}-\lambda_{d}^{-}\right) T_{d}(q)\right|,
$$

where

$$
T_{d}(q, a)=\sum_{\substack{n_{1} \cdots n_{j} \equiv a(q) \\ n_{1} \cdots n_{j} \equiv 0(d)}} 1
$$

and $T_{d}(q)$ is the same sum but with the condition $n_{1} \cdots n_{j} \equiv a(q)$ replaced by the condition $\left(n_{1} \cdots n_{j}, q\right)=1$. Choosing, say, $D=x^{1 / 2000}$ and, after a dyadic subdivision of the range of the $n_{i}$, applying [4, Lemma 2] with a specific value of $\delta$, (see [8, Theorem 5] for example; this can be improved, most recently using work of Margenstern) we deduce that

$$
\sum_{q \in \mathscr{Q}} \sum_{\substack{d \leq D \\(d, q)=1}}\left|T_{d}(q, a)-\frac{1}{\phi(q)} T_{d}(q)\right| \ll x^{1-\delta}
$$

for some small fixed positive $\delta$.

To estimate the contribution of the latter sum in (4.6), we require the following elementary result.

Lemma 3. Let $d$ be square free and relatively prime to $q$. Then

$$
T_{d}(q)=\frac{\omega(d)}{d}\left(\frac{\phi(q)}{q}\right)^{j} \sum_{n_{1}, \ldots, n_{j}} 1+O\left(\frac{x}{z} \tau^{j+1}(d q)\right),
$$


where $\omega$ is multiplicative, $\omega(p) \leq j$, and the implied constant may depend on $j$.

Proof. We have

$$
T_{d}(q)=\sum_{k \mid d} \mu(k) \sum_{\left(n_{i}, k q\right)=1} 1=\sum_{k \mid d} \mu(k) \sum_{m_{i} \mid k q} \mu\left(m_{1}\right) \cdots \mu\left(m_{j}\right) \sum_{n_{i} \equiv 0\left(m_{i}\right)} 1 .
$$

Now,

$$
\sum_{n_{i} \equiv 0\left(m_{i}\right)} 1=\sum_{\substack{r_{1} \cdots r_{j} \sim x / m_{i} \cdots m_{j} \\ r_{i}>z / m_{i}}} 1=\int_{\underline{(\underline{m})}}+O\left(\frac{x}{z} \frac{\sum_{i} m_{i}}{\prod_{i} m_{i}}\right),
$$

where $\underline{m}=\left(m_{1}, \ldots, m_{j}\right)$ and

$$
\int_{(\underline{m})}=\int_{\substack{u_{i}>z / m_{i} \\ x / 2 m_{1} \cdots m_{j}<u_{1} \cdots u_{j} \leq x / m_{1} \cdots m_{j}}} d \int_{1} \cdots d u_{j} .
$$

By a simple substitution

$$
\int_{(\underline{m})}=\frac{1}{\prod_{i} m_{i}} \int_{(\underline{1})}
$$

where $\underline{1}=(1, \ldots, 1)$ and so

$$
T_{d}(q)=\sum_{k \mid d} \mu(k)\left(\frac{\phi(k)}{k}\right)^{j}\left(\frac{\phi(q)}{q}\right)^{j} \int_{(1)}+O\left(\frac{x}{z} \sum_{k \mid d} \sum_{m_{i} \mid k q} 1\right) .
$$

Since $\int_{(\underline{1})}=\sum_{n_{1}, \ldots, n_{j}} 1+O(x / z)$, we complete the proof of the lemma with

$$
\omega(p)=p\left(1-\left(\frac{p-1}{p}\right)^{j}\right) \leq j .
$$

Recalling that $D=x^{1 / 2000}$ and $z>x^{1 / 7}$, we get, on combining Lemmata 2 and 3 with $\kappa=j$,

$$
\sum_{q \in \mathscr{Q}} \frac{1}{\phi(q)}\left|\sum_{\substack{d \leq D \\(d, q)=1}}\left(\lambda_{d}^{+}-\lambda_{d}^{-}\right) T_{d}(q)\right| \ll x L^{-1} s^{3-s} \sum_{q \in \mathscr{Q}} \frac{1}{\phi(q)}+x^{7 / 8}
$$

and by (4.2) this is easily seen to be $\ll x L^{-1}\left(\theta-\frac{1}{2}\right)^{2} \sum_{q \in \mathscr{Q}}(1 / \phi(q))+x^{1-\delta(A)}$, where $\delta(A)$ may depend on $A_{0}$ and hence on $A$.

Combining this with (4.6) and (4.7) we have

$$
\sum_{q \in \mathscr{Q}}\left|\Delta_{j}(x, w ; q, a)\right| \ll x L^{-1}\left(\theta-\frac{1}{2}\right)^{2} \sum_{q \in \mathscr{Q}} \frac{1}{\phi(q)}+x^{1-\delta(A)} .
$$

Combining (4.8) with (4.3) and (4.5) we get (3.2) for $j=1,2,3$.

We remark that (4.8) is obviously easy to improve but that this bound will in any case occur later (in \$7) in a way that is essential from the point of view of our method. 


\section{The cases $4 \leq j \leq 6$ : A subdivision}

Definition. The sequence $\left(n_{1}, \ldots, n_{j}\right)$ is "exceptional" of type $B_{r}$ if it can be partitioned into $r$ subsets whose products $d_{1}, \ldots, d_{r}, d_{1} \geq d_{2} \geq \cdots \geq d_{r}$, satisfy one of the following:

$\left(\mathrm{B}_{4}\right)$

$\left(\mathrm{B}_{5}\right)$

$\left(\mathrm{B}_{6}\right)$

$$
r=4, \quad d_{1} \leq \Delta d_{2}, \quad d_{3} \leq \Delta d_{4},
$$

$$
r=5, \quad d_{3} \leq \Delta d_{5},
$$$$
r=6, \quad d_{4} \leq \Delta d_{6}
$$

with $\Delta=w^{2}$.

A sequence $\left(n_{1}, \ldots, n_{j}\right)$ is "regular" if it is not exceptional of any type. We may by symmetry restrict to sequences with $n_{1} \geq n_{2} \geq \cdots \geq n_{j}$.

A regular sequence is called "smoothable" if $j=4, n_{1} \geq n_{2} \geq n_{3} \geq n_{4}$, and one of the following holds:

$$
n_{4} n_{1}>x^{1 / 2+6 \eta}
$$

or

$$
\begin{gathered}
x^{1 / 6-\delta}<n_{4}<x^{1 / 6+\delta}, \quad x^{1 / 4-\delta}<n_{3} \leq n_{2}<x^{1 / 4+\delta}, \quad \text { and } \\
x^{1 / 3-\delta}<n_{1}<x^{1 / 3+\delta}
\end{gathered}
$$

with $\delta=10^{-2}$, say, and $\eta=10^{-4}$ as in the introduction.

\section{Regular NONSMOOTHABLE SEQUENCES}

The regular sequences that are not smoothable in the above sense are dealt with by first applying a dyadic subdivision of the variables $n_{i}$ as in $[4, \S 13]$ followed by the application of Theorems 3 and 4 of [4] in precise correspondence with their application in $[4, \S 15]$; in fact, our choice of $\Delta=w^{2}$ is determined by the hypotheses of Theorems 3 and 4 of [4]. (Here we have $V=1, N_{1}^{*}=N_{1}$.) Their contribution $E_{j n}$ to $E_{j}(4 \leq j \leq 6)$ is thus found to satisfy the bound

$$
E_{j n} \ll x L^{-A}
$$

for arbitrary $A$.

\section{THE EXCEPTIONAL SEQUeNCES}

We estimate the contribution, say $E_{j h}$ to $E_{j}(4 \leq j \leq 6)$, of the exceptional sequences of type $B_{r}$. The method is that of [4, $\left.\S 14\right]$ using Theorem 2, Lemmata 14 and 15 of [4]. Following that argument, we now find that

$$
\begin{aligned}
E_{j b} & \ll \frac{x}{\log x}\left(\frac{\log \Delta}{\log x}\right)^{2} \sum_{q \in t_{i}} \frac{1}{\phi(q)}+O\left(x L^{-A}\right) \\
& \leq \frac{x}{\log x}\left(C_{j}\left(\theta-\frac{1}{2}\right)^{2}+O\left(\frac{\log \log x}{\log x}\right)^{2}\right) \sum_{q \in \ell^{\prime}} \frac{1}{\phi(q)}+O\left(x L^{-A}\right)
\end{aligned}
$$


which is precisely sufficient for the main theorem. Here $C_{j}$ is a constant and the first $O$-constant may depend on $A$.

We remark that it is only in the consideration of these exceptional sequences that the first two terms arise. Elsewhere $\left(\theta-\frac{1}{2}\right)^{2}$ can be improved as in $\S 3$, and the second term can be deleted. The assumption that $\mathscr{Q}$ is an interval is used only here and is needed in order to apply Theorem 2 of [4]. Although Theorem 2 of [4] is stated for dyadic intervals only, it holds for an interval [1,Q] by summation over dyadic intervals and then for an arbitrary interval by taking differences of intervals $\left[1, Q^{\prime}\right]$ and $[1, Q]$.

\section{REgUlAR SMOOTHABLE SEQUENCES}

It remains to bound the contribution $E_{4 s}$ to $E_{4}$ of the smoothable regular sequences of $\S 5$. Here, as in $[4, \S 15]$, we make use of Theorem 5 of [4], but as in the cases $1 \leq j \leq 3$, we require greater care with the fundamental lemma.

We consider $\widetilde{\Delta}_{4}(x, z ; q, a)$, which we define to be the contribution to $\Delta_{4}(x, z ; q, a)$ (defined in (4.1)) from those sequences $\left(n_{1}, \ldots, n_{4}\right)$, that are smoothable (see (5.1) and (5.2)). As in $\S 4$, we reduce the problem to the estimation of $\widetilde{\Delta}_{4}(x, w ; q, a)$. Using the Buchstab identity and Theorem 1, we get (compare (4.5)) with an obvious notation

$$
\sum_{q \in \mathscr{Q}}\left|\sum_{w \leq p<z} \tilde{\Delta}_{4 p}(x, p ; q, a)\right| \ll x L^{-A} .
$$

To treat $\widetilde{\Delta}_{4}(x, w ; q, a)$, we apply the fundamental lemma, now with $\kappa=1$. Arguing as in $\S 4$, we have

$$
\begin{aligned}
\left|\widetilde{\Delta}_{4}(x, w ; q, a)\right| \leq & \left|\sum_{(d, q)=1} \lambda_{d}^{+}\left\{U_{d}(q, a)-\frac{1}{\phi(q)} U_{d}(q)\right\}\right| \\
& +\left|\sum_{(d, q)=1} \lambda_{d}^{-}\left\{U_{d}(q, a)-\frac{1}{\phi(q)} U_{d}(q)\right\}\right| \\
& +\frac{1}{\phi(q)}\left|\sum_{(d, q)=1}\left(\lambda_{d}^{+}-\lambda_{d}^{-}\right) U_{d}(q)\right| .
\end{aligned}
$$

Here

$$
U_{d}(q, a)=\sum_{n_{1} \cdots n_{4} \equiv a(q)} 1
$$

restricted to those sequences $\left(n_{1}, \ldots, n_{4}\right)$ satisfying the constraints $n_{1} \geq n_{2} \geq$ $n_{3} \geq n_{4}, n_{1} \equiv 0(d)$ and $\left(n_{2} n_{3} n_{4}, P(w)\right)=1$, while $U_{d}(q)$ is the same sum but with the condition $n_{1} \cdots n_{4} \equiv a(q)$ replaced by $\left(n_{1} \cdots n_{4}, q\right)=1$.

To bound the contribution to $E_{4 s}$ from the first two sums in (8.2), we make a dyadic subdivision of the variables, say, $d \sim D_{1}, n_{i} \sim N_{i}(1 \leq i \leq 4)$. We choose, say, $D=x^{1 / 2000}$ as in $\S 4$. To each box having contributions satisyfing 
(5.1), we may apply Theorem 5 of [4] with $L=N_{1} / D_{1}, K=N_{4} D_{1}, M=$ $N_{2} N_{3}$. To other boxes with nonempty contributions (so (5.2) is satisfied), we apply Theorem 5 of [4] with $L=N_{1} / D_{1}, K=N_{3} D_{1}, M=N_{2} N_{4}$. These yield

$$
\sum_{q \in \mathscr{Q}}\left|\sum_{\substack{(d, q)=1 \\ d \leq D}} \lambda_{d}^{ \pm}\left\{U_{d}(q, a)-\frac{1}{\phi(q)} U_{d}(q)\right\}\right| \ll x L^{-A} .
$$

To bound the contribution from the last sum in (8.2), we need an estimate for $U_{d}(q)$. This corresponds now, however, to the easiest case, $j=1$ of Lemma 3 , in that we can fix $n_{2}, n_{3}, n_{4}$ and sum over $n_{i} \equiv 0(d)$. In this way, we get, since $n_{1}>x^{1 / 4} / 2$,

$$
U_{d}(q)=U_{1}(q)+O\left(x^{3 / 4} L^{2} \tau(q)\right) .
$$

Combining this with Lemma 2 , we get

$$
\sum_{q \in \mathscr{Q}} \frac{1}{\phi(q)}\left|\sum_{(d, q)=1}\left(\lambda_{d}^{+}-\lambda_{d}^{-}\right) U_{d}(q)\right| \ll \frac{x}{\log x} s^{1-s} \sum_{q \in \mathscr{Q}} \frac{1}{\phi(q)}+x^{4 / 5} .
$$

Combining the Buchstab identity and (8.1)-(8.4), we get for $E_{4 s}$ the same bound as for $E_{j}, 1 \leq j \leq 3$.

The results of $\S \S 4-8$ now combine to complete the proof of the main theorem.

\section{REFERENCES}

1. A. Balog, $p+a$ without large prime factors, Sém. Théor. Nombres, Bordeaux, Exposé 31, 1983-84.

2. E. Bombieri, On the large sieve, Mathematika 12 (1965), 201-225.

3. E. Bombieri, J. B. Friedlander, and $\mathrm{H}$. Iwaniec, Primes in arithmetic progressions to large moduli, Acta Math. 156 (1986), 203-251.

4. __ Primes in arithmetic progressions to large moduli. II, Math. Ann. 277 (1987), 361-393.

5. E. Fouvry, Théorème de Brun-Titchmarsh; application au théorème de Fermat, Invent. Math. 79 (1985), 383-407.

6. E. Fouvry and F. Grupp, On the switching principle in sieve theory, J. Reine Angew. Math. 370 (1986), 101-126.

7. J. Friedlander and H. Iwaniec, On Bombieri's asymptotic sieve, Ann. Scuola Norm. Sup. Pisa Cl. Sci. (4) 5 (1978), 719-756.

8. Incomplete Kloosterman sums and a divisor problem, Ann. of Math. (2) 121 (1985), 319-350.

9. H. Iwaniec, Rosser's sieve, Acta Arith. 36 (1980), 171-202.

10. Yu. V. Linnik, The dispersion method in binary additive problems, Trans. Math. Mono., Vol. 4, Amer. Math. Soc., Providence, RI, 1963

11. B. Rousselet, Inégalités de type Brun-Titchmarsh en moyenne, Groupe de Travail en Théorie Analytique des Nombres, Orsay (to appear).

Institute for Advanced Study, Princeton, NJ 08540

Scarborough College, University of Toronto, Scarborough, Ontario, Canada M1C 1A4

Department of Mathematics, Rutgers University, New Brunswick, NJ 08903 